\title{
Study on Microcalcification Detection Using Wavelet Singularity
}

\author{
Guo Jinghuan ${ }^{1}$, Chen Shenglai ${ }^{2}, \mathrm{Ge} \mathrm{Ku}^{3}$, Sun Zhaoqian ${ }^{4}$ \\ ${ }^{1}$ Institute of Information Sciences and Technology, Dalian Maritime University \\ ${ }^{2}$ The 28th Research Institute of China Electronics Technology Group Corporation \\ ${ }^{3}$ Institute of Information Sciences and Technology, Dalian Maritime University \\ ${ }^{4}$ Institute of Information Sciences and Technology, Dalian Maritime University \\ 'guojh1974@126.com, ${ }^{2}$ chensl_01@163.com, ${ }^{3}$ gek88@163.com, \\ 4sunzhaoqian123@126.com
}

\begin{abstract}
A microcalcification detection method based on wavelet singularity was presented because of microcalcification singularity characteristic. Firstly, the source image is decomposed in multi-scales wavelet coefficients. Secondly, coefficients in low-pass band are removed and coefficients in high-pass band are enhanced contrast by nonlinear method. Lastly, fisher discriminant was adopted in segment microcalcifications. Experiment results showed that wavelet basis with shorter support and lower regularity is more sensitive to noise, while wavelet basis with longer support, higher regularity and higher order vanishing moment could segment indistinct microcalcifications, but sometime could not segment small microcalcifications. The results also showed the detect effect DAUB4 wavelet is best and its detection ratio is about $96 \%$.
\end{abstract}

Keywords: microcalcification detection; wavelet singularity; wavelet basis; Fisher discriminant

\section{Foreword}

The film of X-Ray, the important method in the field of breast carcinoma, is provided with the merit of high resolution and visible microcalcification structure. Now, the microcalcification detection is one of the popular topics in the field of Medical image analysis and recognition $[1,2]$, but it is very difficult to be carried out because of its tiny, singularity and distributional difference.

In recent years, wavelet transform is widely used in the field of image analysis and recognition such as in the detect microcalcification because of good time-frequency localization properties [3]. But we are unable to make full use of the advantage of wavelet transform to the fields. A microcalcification detection method based on wavelet singularity was presented by the Gurcans [4]. The method is to decompose the source image in multi-scales wavelet coefficients, and select one of the multi-scales wavelet coefficients to count the value of the local skewness and kurtosis. The point that the value of the local skewness and kurtosis is bigger than the threshold value is called as the microcalcification. Because of microcalcification singularity complexity, the microcalcification is segmented difficultly only by the skewness and kurtosis of wavelet. A method based on the high frequency information of the wavelet is presented by the Ted C. Wangs [5]. Firstly, the method is to decompose the source image to the coefficients in low-pass band and the coefficients in high-pass band. Secondly, the wavelet is reconfigured based on the coefficients in high-pass band. Lastly, the segmentation is adopted in segmenting microcalcifications. The fluency of the high-frequency voice and the the vessel make the method not make full use of the singularity characteristic to segment microcalcifications. 
A microcalcification detection method based on wavelet singularity was presented because of microcalcification singularity characteristic, similarly to the singular point of a functions or the signal peak.

\section{Theory of Wavelet Singularity}

\subsection{Signal Singularity}

In mathematics, the local singularity of function is represented by the Lipschitz exponent. The wavelet theory shows: the rule that amplitude varies along with the maximum of module is decided by the Lipschitz exponent of the signal jump point [6].

$$
\left|f\left(x_{0}+h\right)-P_{n}\left(x_{0}+h\right)\right| \leq A|h|^{2}
$$

Definition 1: $n$ is supposed as a non-negative integer, $n<a \leq n+1$ If there are two constants $A \& h>0$ and polynomial $P_{n}(h)$ with $n$ rank, the inequality of $\left|f\left(x_{0}+h\right)-P_{n}\left(x_{0}+h\right)\right| \leq A|h|^{2}$ is tenable, so the Lipschitz exponent of $f(x)$ at the point of $x_{0}$ is the number of $a$.

The magnitude of the singularity is characterized by the Lipschitz exponent at some point of the function, the more the value of $a$, the higher the smoothness, the bigger the singularity. If the function of $f(x)$ is differentiable at some point, $a \geq 1$. If the function of $f(x)$ is discontinuities and the its value is limited, $0 \leq a \leq 1$. So the function is singularity at the point. To step function, $a=0$ and impulse function, $a=-1$, to white noise, $a=-1 / 2-\varepsilon, \varepsilon>0$.

It was proved by Mallat that wavelet transformation could detect the signal singularity [7]. The maximum of wavelet exponent module corresponds to the signal jump point or edge. The amplitude of the maximum of wavelet exponent module changed along with the change of the image scale, which rule was decided by the local signal singularity of the signal jump point. At the point that the singularity value is greater than zero, the maximum of wavelet transformation module corresponds would get bigger with the increasement of the image scale. It was on the contrary at the point that the singularity value is less than zero. At the point that the singularity value equaled to zero for a step function, the maximum of wavelet transformation module does not change along with the image scale change. So in large image, the wavelet transformation smoothed the noise to make wavelet exponent less, and to improve signal to noise ratio.

\subsection{Choice of Wavelet Basic Function}

The different wavelet basic function can influence the signal singularity detection, and directly influence the effect of microcalcification detection. At present, there is not a unified method to selecting wavelet basic function. It is general way to choose a basic function by the characteristic of wavelet basic function. The characteristic is showed as below:

1) Vanishing moment: If the singularity of the detected signal is $n$, wavelet of $n+1$ step vanishing moment is needed.

2) Compactly support: The length of wavelet compactly support decides the good or bad of signal characteristic. The shorter the compactly support, the more the time-frequency characteristics, and it is advantageous to instant detection of signal.

3) Regularity: Generally, the smoothness of the wavelet basic is represented by regularity. The higher regularity, the well the smoothness. Usually, smooth signal is analyzed by the wavelet basic with higher-regularity and mutation signal by lower-regularity. 
Generally, vanishing moment, compactly support and regularity are closely correlated. The higher the regularity, the higher order vanishing moment, and the longer the support. Because the microcalcification singularity characteristic is similar to mutation signal, we should choose the wavelet basic with the shorter support, the proper vanishing moment and regularity.

\section{Enhanced Wavelet Coefficient}

\subsection{D Image Wavelet Transform}

Supposed, $\phi(x, y)$ represents two-dimensional function, $\varphi^{H}, \varphi^{V}, \varphi^{D}$ respectively represent the horizontal, the vertical and the diagonal in the two-dimensional wavelet, which have the characteristic as below:

$$
\left\{\begin{array}{l}
\phi_{j, m, n}(x, y)=2^{-j} \phi\left(2^{-j} x-m, 2^{-j} y-n\right) \\
\varphi_{j, m, n}^{i}(x, y)=2^{-j} \varphi^{i}\left(2^{-j} x-m, 2^{-j} y-n\right), i=\{H, V, D\}
\end{array}\right.
$$

So, wavelet decomposition of the two-dimension image function $f(x, y)$ with $M \times N$ $f(x, y)$ is represented by:

$$
\left\{\begin{array}{l}
W_{\phi}(j, m, n)=\frac{1}{\sqrt{M N}} \sum_{x=0}^{M-1} \sum_{y=0}^{N-1} f(x, y) \phi_{j, m, n}(x, y) \\
W_{\varphi}^{(i)}(j, m, n)=\frac{1}{\sqrt{M N}} \sum_{x=0}^{M-1} \sum_{y=0}^{N-1} f(x, y) \varphi_{j, m, n}(x, y) ; i=\{H, V, D\}
\end{array}\right.
$$

In which, $W_{\phi}$ represents low pass approximations coefficient, $W^{H}, W^{V}, W^{D}$ respectively represent the horizontal, the vertical and the diagonal high frequency details, $j$ represents resolution.

Two-dimension discrete wavelet is decomposed by digital filter and down sampling by 2 -factor. The schematic diagram for image decomposition is showed in the figure 1 , in which, $g$ represented low-pass filter, $h$ represents high-pass filter, $\downarrow 2$ represented down-sampling by 2 factor.

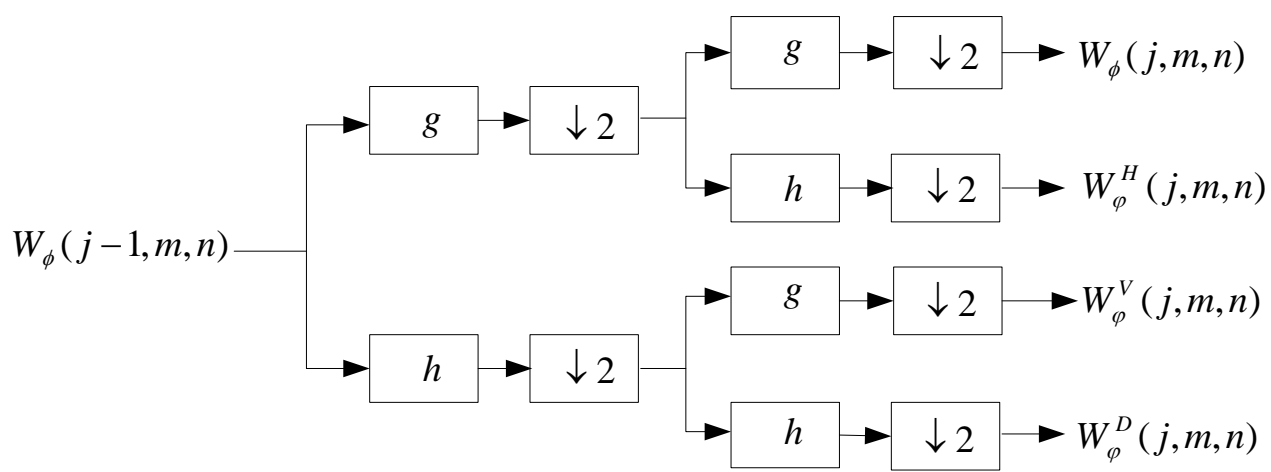

Figure 1. Wavelet Decomposition with Two-Dimension Image

\subsection{Enhanced Wavelet Coefficient}

The result of three level wavelet transform was showed in Figure 1. The brighter point was the microcalcification singularity, indicating that wavelet coefficient of the 
microcalcification singularity was bigger than the background, but the contrast was not enough strong, and was improved by enhanced wavelet coefficient. The function expression to enhance high frequency coefficient was showed as below:

$$
f^{\prime}(x, y)= \begin{cases}0 & |f(x, y)| \leq c \\ a \cdot(f(x, y)-c)^{2} & f(x, y)>c \\ a \cdot(f(x, y)+c)^{2} & f(x, y)<-c\end{cases}
$$

In which, $f(x, y)$ is wavelet coefficient before transformed, and $f^{\prime}(x, y)$ is the coefficient after transformed. $a$ is enhanced coefficient. The different enhanced coefficient is chose with different wavelet sub-bands. $c$ is the segmentation, and it is chose by image characteristic and usually is the amplitude average value of wavelet coefficient.

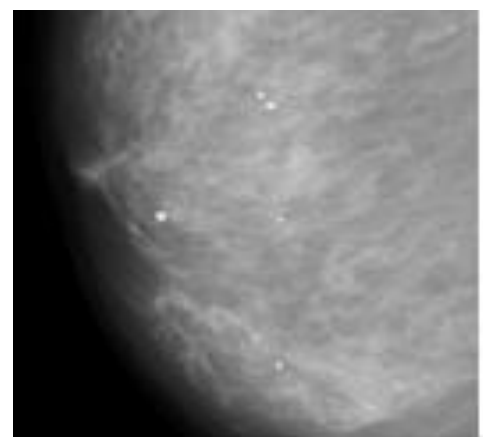

a Original image

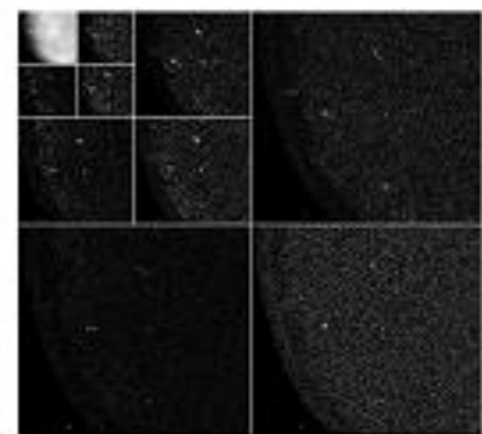

b Wavelet Analytical Image

\section{Figure 2. Result of Wavelet Analysis}

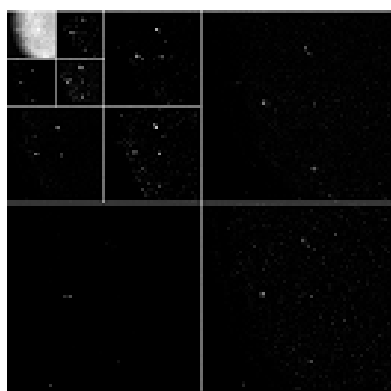

Figure 3. Enhanced Wavelet Image

The analyzed image transformed by enhanced wavelet coefficient is showed in Figure 3 . The image contrast has been improved, and wavelet coefficient of background noise is well restrained.

\section{Microcalcification Segment Discriminant Based on Fisher}

$W_{\phi}$, the low pass approximations coefficient of image function $f(x, y)$, is called background information, it can be set zero. $W_{\varphi}^{(i)}(j, m, n)$ represents the high frequency information including microcalcifications. Because of microcalcification singularity characteristic, wavelet coefficient is bigger. The wavelet coefficient can be separate by setting threshold. Fisher threshold is adopted to separate the microcalcifications wavelet coefficient in the paper.

Fisher discriminant is designed to seek for an optimum differentiating direction. Different samples were projected to the direction and separated as possible, and the same 
class samples can be massed. The Fisher discriminant theoretically is the best way to differentiate the degree of separation [8].

The computation method of Fisher discriminant is that wavelet coefficient $X=\left\{x_{1}, x_{2}, \cdots, x_{N}\right\}$ is divided into the background $\omega_{1}$ and the microcalcifications $\omega_{2}$. The number of the background is $d_{1}$, the microcalcifications coefficient is $d_{2}$, and supposed that $W$ is the best differentiating direction. So,

$$
Y=W^{T} X
$$

Supposed, the samples average value $m_{i}$, dispersion within class $S_{i}$, the general class dispersion $S_{w}$, inter-class dispersion $S_{b}$ are respectively defined as

$$
\begin{aligned}
& m_{i}=\frac{1}{d_{i}} \sum_{x \in \omega_{i}} x ; i=1,2 \\
& S_{i}=\sum_{x \in \omega_{i}}\left(x-m_{i}\right)\left(x-m_{i}\right)^{T} ; i=1,2 \\
& S_{w}=S_{1}+S_{2} \\
& S_{b}=\left(m_{1}-m_{2}\right)\left(m_{1}-m_{2}\right)^{T}
\end{aligned}
$$

When X was projected to the direction of $W, \tilde{m}_{i}, \tilde{S}_{i}, \tilde{S}_{w}$ respectively were:

$$
\begin{aligned}
& \tilde{m}_{i}=\frac{1}{d_{i}} \sum_{y \in \omega_{i}} y ; i=1,2 \\
& \tilde{S}_{i}=\sum_{y \in \omega_{i}}\left(y-\tilde{m}_{i}\right)\left(y-\tilde{m}_{i}\right)^{T} ; i=1,2 \\
& \tilde{S}_{w}=\tilde{S}_{1}+\tilde{S}_{2}
\end{aligned}
$$

After projected, it is expected that the difference of average value $\tilde{m}_{1}-\tilde{m}_{2}$ is enough larger, and all kinds of samples are expected to be more concentrated. Namely, the general class dispersion is the better the less. So it is defined that:

$$
J(W)=\frac{\tilde{\left(m_{1}-\tilde{m}_{2}\right)^{2}}}{\tilde{S}_{1}^{2}+\tilde{S}_{2}^{2}}
$$

From the above, when the dispersion is the minimum and the average difference is the maximum, $J(W)$ would get the maximum value, then the obtained direction of $W^{*}$ is the most optimum. The projection relationship is:

$$
\begin{aligned}
& \tilde{\left(m_{1}-\tilde{m_{2}}\right)^{2}}=\left(W^{T} m_{1}-W^{T} m_{2}\right)^{2}=W^{T}\left(m_{1}-m_{2}\right)\left(m_{1}-m_{2}\right)^{T} W=W^{T} S_{b} W \\
& \tilde{S}_{i}=\sum_{x \in \omega_{i}}\left(W^{T} x-W^{T} m_{i}\right)^{2}=W^{T}\left[\sum_{x \in \omega_{i}}\left(x-m_{i}\right)\left(x-m_{i}\right)^{T}\right] W=W^{T} S_{i} W
\end{aligned}
$$




$$
\begin{aligned}
& \tilde{S}_{1}^{2}+\tilde{S}_{2}^{2}=W^{T}\left(S_{1}+S_{2}\right) W=W^{T} S_{w} W \\
& J(W)=\frac{W^{T} S_{b} W}{W^{T} S_{w} W}
\end{aligned}
$$

$J(W)$ is a general Rayleigh entropy, and after the most optimum projection direction $W^{*}$ is gained by Lagrange parameters, the samples are classified in the space of $Y$.

\section{Experimental Result}

The method mentioned in the paper was proved by MIAS, which included 322 clinically verified X-ray images, the image is $1024 \times 1024$. In experiment the 151 microcalcifications from 25 images were used as experimental subjects.

The fast algorithm of Mallat was adopted under experiment environment of Windows XP with Dual-Core 2.60GHz CPU and 2G, using developing tool of VC6.0, HAAR、 DAUB4、DAUB8、SYM4、SYM8 were used as wavelet basic. The characteristic was showed in the below table. The rank of wavelet basic was 3, using relevance ratio TP and the error rate EP to detect the ability of wavelet basic. The experiment result was showed Figure 4-Figure 6 and Table 2, in which the result was marked by the red box. Based on the result of Figure 4, wavelet basic could efficiently detect the microcalcification with obvious characteristic, but wavelet basic with shorter support and lower regularity was more sensitive to noise and the edge of image. So the false positive objects of HAAR were more than that of wavelet basic else. The result of Figure 5 showed that wavelet basic of HAAR、DAUB4、DAUB8 with shorter support, lower regularity and lower order vanishing moment could not efficiently detect the microcalcification with obscure characteristic. But wavelet basic of DAUB8 and SYM8 could completely the microcalcification. The result of Figure 6 showed that wavelet basic could not detect all of the microcalcification, especially to the small microcalcification, it was more difficult to be detected. The general result was showed in the Table 2, and wavelet basic of DAUB4 was the best. The fallout ratio of HAAR was high. From the detection of speed, the detecting speed of wavelet basic with the same longer support was equivalent, but the one with longer support was slower.

Table 1. Characteristic of Wavelet Basic

\begin{tabular}{c|c|c|c}
\hline Wavelet basic & Long support & Vanishing moment & Regularity \\
\hline HAAR & 1 & 1 & Discontinuous, No regularity \\
\hline DAUB4 & 7 & 4 & 0.8 \\
\hline DAUB8 & 15 & 8 & 1.6 \\
\hline SYM4 & 7 & 4 & 0.8 \\
\hline SYM8 & 15 & 8 & 1.6 \\
\hline
\end{tabular}

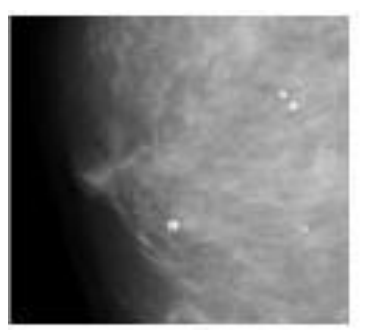

a. Original image

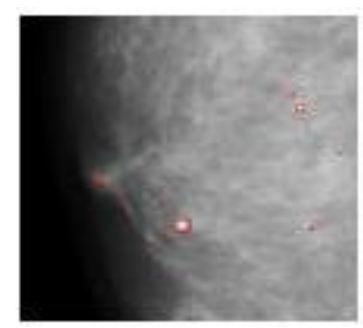

b. HAAR result

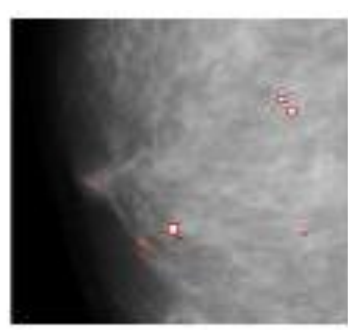

c. DAUB4 result

(White point is microcalcification) 


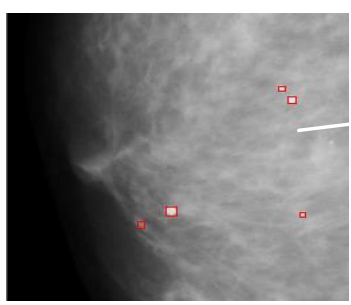

d. DAUB8 Detect Result

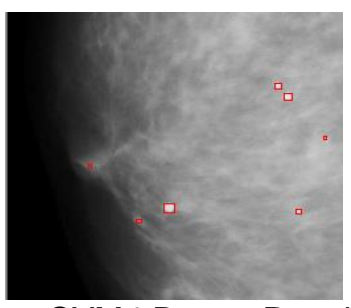

e. SYM4 Detect Result

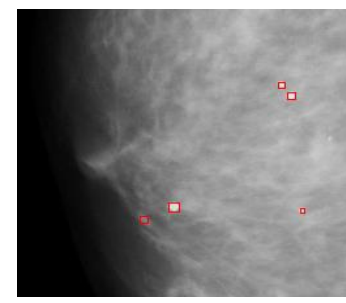

f. SYM8 Detect Result

Figure 4. Detect Result of Original Image Using Different Wavelet Filter

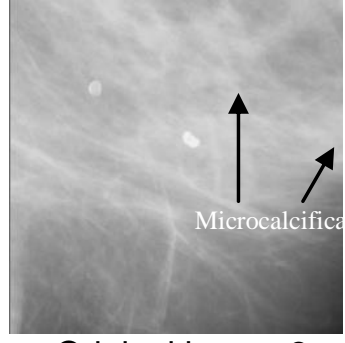

a. Original image 2

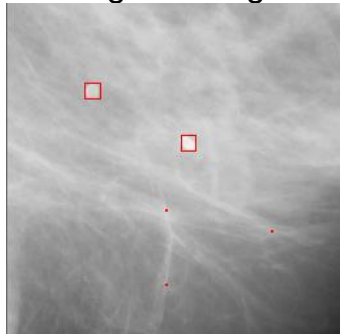

d. DAUB8 detect result

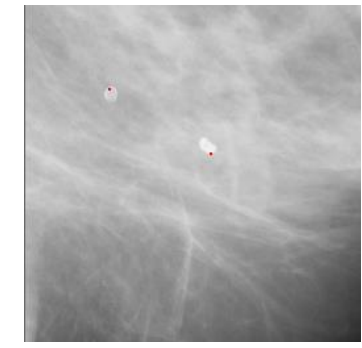

b. HAAR detect result

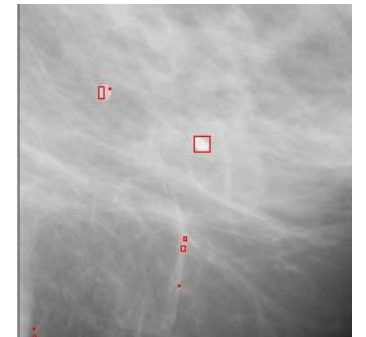

e. SYM4 detect result

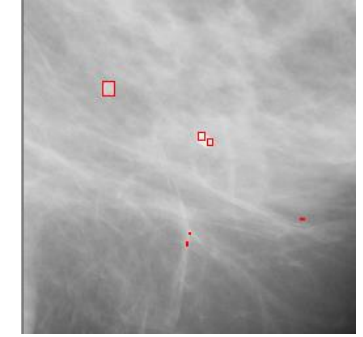

c. DAUB4 detect result

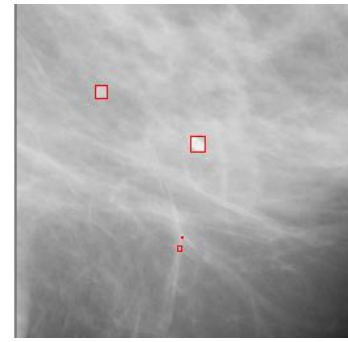

f. SYM8 detect result

Figure 5. Detect Result of Originalimage 2 using Different Wavelet Filter
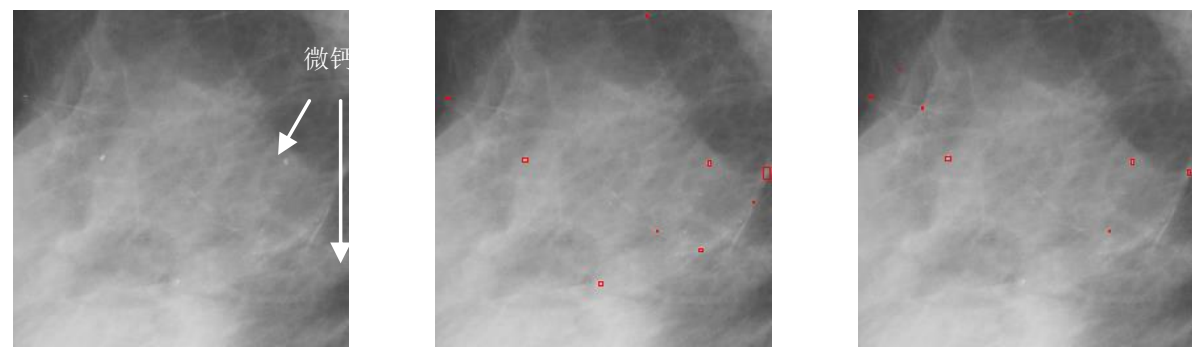

a. Original Image 3

b. SYM4 Detect Result

c. SYM8 Detect Result

Figure 6. Detect Result of Original Image 3 using Different Wavelet Filter

Table 2. General Detect Result of Different Wavelet Basic

\begin{tabular}{c|c|c|c}
\hline Wavelet basic & relevance ratio TP & $\begin{array}{c}\text { Error rate } \\
\text { EP }\end{array}$ & $\begin{array}{c}\text { Average detect } \\
\text { time(ms) }\end{array}$ \\
\hline HAAR & $90.07 \%$ & $75.4 \%$ & 1578 \\
\hline DAUB4 & $96.03 \%$ & $68.3 \%$ & 1813 \\
\hline DAUB8 & $93.38 \%$ & $65.1 \%$ & 2328 \\
\hline SYM4 & $95.36 \%$ & $67.8 \%$ & 1823 \\
\hline SYM8 & $94.04 \%$ & $64.8 \%$ & 2335 \\
\hline
\end{tabular}

During experiment, the method motioned in the paper was contrasted with the morphology detect method by the one the J.C. Fu's [9]. Firstly the Top-hat operator is adopted to do preliminary detection for the image microcalcification. Secondly, the edge of microcalcification is positioned by the edge detection operator of Sobel and Canny. Lastly, closed operator is used in noise filtering. The morphology detect result was 
showed in Table 3. Contrasted with Table 2, the relevance ratio of the paper provided was higher efficiency. The morphology closed operator was adopted, the error rate was lower and the detect speed was higher.

Table 3. Detect result of the Morphology

\begin{tabular}{c|c|c}
\hline relevance ratio TP & Error rate EP & Average detect time(ms) \\
\hline $89.4 \%$ & $16.6 \%$ & 578 \\
\hline
\end{tabular}

\section{Concluding Remarks}

The computer detect method of the image microcalcification is widely used in the field of medical image processing. How to effectively detect the micocalcification is very important significance to subsequent detect in the diagnosis of breast cancer. Microcalcification detect method based on wavelet singularity was presented because the microcalcification is tiny, and the contrast of background is small. A choosing method was studied in the paper. Experiment results showed that the synthetic detection effectiveness of DAUB4 was the best. The wavelet transform method was contrasted with the morphology detect method, and it was proved that the wavelet transform method was more effective in the relevance ratio. But because the false positive object was too much, the artificial intelligence method, such as SVM and neural network, must be used to further detect the false positive object.

\section{Acknowledgements}

Study on CT Image Automatic Segment based on Machine Learning ,Young Key Teachers Foundation Projects of Dalian Maritime University (2012QN031)

Study on network technology of intelligent navigation, Fundamental Research Funds for the Central University (3132013308)

\section{References}

[1] S. M. Astley and F. J. Gilbert, "Computer-aided detection in mammography", Clinical Radiology, vol. 59, no. 5, (2004), pp. 390-399.

[2] L. Shunan, W. Baikun, M. Zhenhe and W. Ruiping, "A Novel ROI Extracting Technique Based on Wavelet Transform for the Detection of Micro-calcifications in Mammograms", Journal of Biomedical Engineering, vol. 22, no. 2, (2005), pp. 360-362.

[3] I. Daubechies, "The wavelet transform time-frequency localization and signal analysis", IEEE Trans on Information Theory, vol. 36, no. 5, (1990), pp. 961-1004.

[4] M. Nafi, Gurcan, "Detection of microcalcifications in mammograms using higher order statistics", IEEE Signal Processing Letters, vol. 4, no. 8, (1997), pp. 213-216.

[5] T. C. Wang and N. B. Karayiannis, "Detection of microcalcifications in digital mammograms", IEEE Trans on Medical Imaging, vol. 17, no. 4, (1998), pp. 498-509.

[6] S. G. Mallat and W. L. Hwang, "Singularity detection and processing with wavelets", IEEE Trans on Information Theory, vol. 38, no. 2, (1992), pp. 617-643.

[7] S. Mallat and S. Zhong, "Characterization of signals from multiscale edges", IEEE Trans on Pattern Analysis and Machine Intelligence, vol. 14, no. 7, (1992), pp. 710-732.

[8] B. Zhaoqi and Z. Xuegong, Pattern Recognition (second edition) Tsinghua University press, (2002), pp. 87-90.

[9] J. C. Fu, S. K. Lee and S. T. C. Wong, "Image segmentation feature selection and pattern classification for mammographic microcalcifications", Computerized Medical Imaging and Graphics, vol. 29, no. 6, (2005), pp. 419-429. 


\section{Authors}

Guo Jinghuan, (1974), female, Liaoning Changtu Country, Doctor, Associate Professor. Research on Image Processing and Pattern Recognition.

Chen Shenglai, (1978), male, Zhejiang Haining Country, Senior Engineer. Research on Image Processing and Intelligence Fusion.

Ge Kun, (1989), male, Shandong Heze, Master in reading, Research on Web Service Somposition and Development of Agent Aystem based on JADE.

Sun Zhaoqian, (1990-), female, Shandong Jining, Master in reading, Research on Software Test and Software Fault location. 
International Journal of Signal Processing, Image Processing and Pattern Recognition Vol.7, No.1 (2014) 